\title{
07 | De la intuición a la metodología. \\ Propedéutica del proyectar en el curso básico de la HfG Ulm
} _Ángel Luis Fernández Campos, Emilia Benito Roldán y María Dolores Sánchez Moya

\section{"Querido Gyorgy Kepes,}

como probablemente sepa, estoy organizando un instituto que continúa nuestra querida vieja Bauhaus en Alemania. Naturalmente es algo diferente que usted encontrará adjunto en un folleto preliminar.

Esta carta es para informarle que necesitaríamos para un primer año algunos profesores de prestigio. Tenemos buenos jóvenes artistas pero no están formados como docentes. Nuestros cursos básicos necesitan profesores invitados para instruir a pequeños grupos de estudiantes y al mismo tiempo a nuestros jóvenes.

Para esta labor también he convocado a venir desde los Estados Unidos a mi viejo amigo Josef Albers. También planeamos llamar a Walter Peterhans. Y ahora quiero preguntarle si podría serle posible venir por algunas semanas o meses y en qué momento."1

\section{"Querido señor Bill,}

Muchas gracias por su carta. Estuvo bien saber acerca de usted y de sus emocionantes planes. Pienso que es una tarea fundamental restablecer la Bauhaus en Alemania, y me complace que usted sea el espíritu que lo guía."

El intercambio de palabras entre un director que no culmina la trayectoria de la Hochschule für Gestaltung y un candidato a profesor que nunca llega a concretarse ${ }^{3}$, expresan la intensidad de la puesta en marcha de la institución dedicada a la enseñanza del proyectar con más influencia en Europa después de la Bauhaus ${ }^{4}$. La escuela siempre se caracterizó por crecer en un estado de ósmosis constante entre un cuerpo docente formado por artistas, científicos, técnicos, arquitectos y teóricos, y un conjunto de estudiantes que acudieron desde todas partes del mundo.

El devenir de la escuela estuvo marcado por una sucesión de intercambios y relevos generacionales e intelectuales con diferentes aproximaciones al proyecto. Convulsos giros pedagógicos determinaron que la institución, que había nacido como una propuesta para la refundación de la Bauhaus, terminara transformándose en el referente europeo de la metodología científica del proyecto en tan solo 15 años. Estos profundos cambios que experimentó la escuela en cuanto a la aproximación al proyecto se manifiestan de forma más significativa en el curso básico.

El curso básico, o Grundkurs, se concibe como un conjunto de enseñanzas de carácter propedéutico o preparatorio que proporcionaba una base común a los alumnos procedentes de varios países y desde distintas ramas de la enseñanza o del mundo profesional, artístico, técnico o artesanal. El paso por este primer curso orientaba a aquellos que lo superaban a continuar sus estudios en los departamentos de diseño industrial, comunicación visual, arquitectura e información ${ }^{5}$. El curso básico se organizaba en cuatro grandes grupos de disciplinas: el Grundlehre, también denominado Visuelle Einführung -aprendizaje del lenguaje visual-, el bloque llamado Darstellungstechniken o técnicas de representación, las ciencias exactas y las asignaturas teóricas. El Visuelle Einführung constituía el núcleo fundamental y el centro de gravedad del Grundkurs, donde se establecían las bases y las enseñanzas fundamentales del proyecto para los futuros diseñadores. El resto de asignaturas tenían un carácter de apoyo técnico -bien de representación, elaboración de modelos en taller-y teórico.

Anthony Froshaug ${ }^{6}$ explica que más que de una evolución, habría que hablar de una sucesión de varios cursos básicos, debido a que las diferencias entre sus contenidos y sus protagonistas fueron muy marcadas ${ }^{7}$. ¿Por qué varios? Son los protagonistas y sus diferentes enfoques del proyecto los que operan las transformaciones de un curso básico que nace bajo la influencia de los Bauhäusler y se extingue como departamento independiente y común, durante el curso 1960-61 tras experimentar una radicalización en la aplicación de la metodología científica en el proyecto. Un recorrido a través de estas enseñanzas permitirá identificar dónde se
Resumen pág 44 | Bibliografía pág 51

Ángel Luis Fernández Campos es Arquitecto titulado en la ETSAM y doctorando en la misma escuela. Está desarrollando una tesis titulada Atlas propedéutico, que aborda la didáctica de las disciplinas visuales de la HfG Ulm y las vanguardias precedentes. Ejerce la docencia como profesor de Educación Plástica y Visual en la enseñanza secundaria pública.

Emilia Benito Roldán es Arquitecto ETSAM y doctorando en la misma escuela. Desarrolla, en el departamento de Composición, una tesis titulada La geometría como lenguaje de las formas. Hermann von Baravalle en la HfG de UIm, que profundiza en la participación de la geometría dinámica en el proceso de proyecto en la HfG UIm. Es profesora de Geometría y Dibujo en la Escuela de Arquitectura de Toledo, de la Universidad de Castilla-La Mancha.

María Dolores Sánchez Moya es Doctor Arquitecto por la UPM (2012) y subdirectora de la Escuela de Arquitectura de la Universidad de Castilla-La Mancha, donde además imparte docencia de Proyectos y Composición. Ha realizado una estancia de investigación post doctoral en el archivo de la HfG Ulm, desarrollando junto a Ángel Luis Fernández Campos y Emilia Benito Roldán una investigación sobre el curso básico de esta escuela.

\section{Palabras clave}

Propedéutica, proyectar, curso básico, grundlehre, Hochschule für Gestaltung UIm, Max Bill, Tomás Maldonado, Hermann von Baravalle, Horst Rittel, metodología 
${ }^{4}$ La escuela de Ulm nace vinculada al movimiento de la resistencia anti nazi. Sophie y Hans Scholl, dos estudiantes de la universidad de Múnich, fueron dirigentes y activistas del grupo de resistencia no violenta la Rosa

Blanca. Este grupo, de convicciones cristinas, se oponía al régimen de Hitler, no solo por motivos politicos sino fundamentalmente por cuestiones éticas y morales. Acusados de tra ción, los hermanos Scholl fueron ejecutados en 1943 y se convirtieron posteriormente en símbolos de la lucha antifascista. Su hermana, Inge Scholl, junto con su marido, Otl Aicher, fueron dos de los fundadores de la escuela de Ulm y principales ideólogos de la escuela en sus inicios.

${ }^{5}$ Los alumnos, al finalizar el curso básico, eran examinados por un claustro de profesores que decidia si habían alcanzado el nivel suficiente para ingresar en los departamentos en el curso siguiente. Los cuatro departamentos de la $\mathrm{HfG}$ evolucionaron a lo largo de la vida de la escuela y en paralelo a la definición de su carácter. Por ejemplo, el departamento de arquitectura y ciudad inicial terminó denominándose departamento de construcción industrializada.

${ }^{6}$ “Es imposible describir el curso fundacional de la escuela de Ulm. Todo lo que puede describirse son una sucesión de varios cursos entre los años 1953-4, cuando la escuela comenzó, y 1960-61 después del cual el curso básico fue abolido". FROSHAUG, Anthony. "Foundation Course, Ulm 1953-61". En: KINROSS, Robin (ed.). Anthony Froshaug. Typography \& texts. Londres: Hyphen press, 2000, p. 133

${ }^{7}$ Anthony Froshaug era diseñador gráfico del Royal College of Arts de Londres y profeso invitado desde 1957 en la HfG. Cuando la escuela comienza, entra en contacto con Max Bill porque sabe de su participación en la publicación de las obras completas de L Corbusier y le propone realizar una versión en inglés. Max Bill le invita a formar parte de la escuela en un primer momento, pero poco después se arrepiente de su decisión al ver la edición en inglés del artículo de Bill "The Bauhaus idea: from Weimar to Ulm" que Froshaug habia realizado en el anuario Architects' Year Book 5 de 1953. Finalmente, es Maldonado quien, en 1957, viaja a Londres para propone formalmente la incorporación de Froshaug a la HfG.

${ }^{8}$ “En aquellos tiempos no habia una idea clara respecto de la profesión que más tarde sería denominada como "diseño industrial". En los paises de habla alemana, era predominante el término "Formgeber" ("dador de forma"). Tampoco había ninguna concepción de la profesión que ahora llamamos "diseño de información" -se hablaba de "gráfica comercial" y "gráfica aplicada". BONSIEPE, Gui. "Sobre la relevancia de la HfG Ulm", en: VV.AA. Ulmer modelle-Modelle nach ulm, hochschule für gestaltung ulm 1953-1968. Ostfildern-Ruit: Hatje Cantz Verlag, 2003.

${ }^{9}$ La escuela en el año de su fundación aún no tenía sede y se alojó durante el primer curso y medio en la Ulm Volkshochschule. La Ulm creada por Inge Scholl y Otl Aicher tras el fin de la II Guerra Mundial, con el apoyo de gobierno militar americano. La escuela proporcionaba educación para adultos por las tardes que incluía conferencias.

${ }^{10}$ Visual training es un curso que sirve para entrenar el ojo y el sentido del diseño y para alentar la apreciación estética del mundo de las proporciones, las formas, los colores, las texturas y los espacios. El curso visual training ideado por Walter Peterhans todavia hoy se imparte en el IIT de Chicago. Los únicos trabajos dirigidos por el propio Peterhans se conservan en el archivo de la HfG Ulm.
[1] Primer ejercicio del Visual Training impartido por Walter Peterhans.

"Ponderar una franja de super

de la misma altura pero distinto ancho integrando los vacíos". Alumno Christopher Naske, Agosto 1953. Fuente: Archivo HfG Ulm.

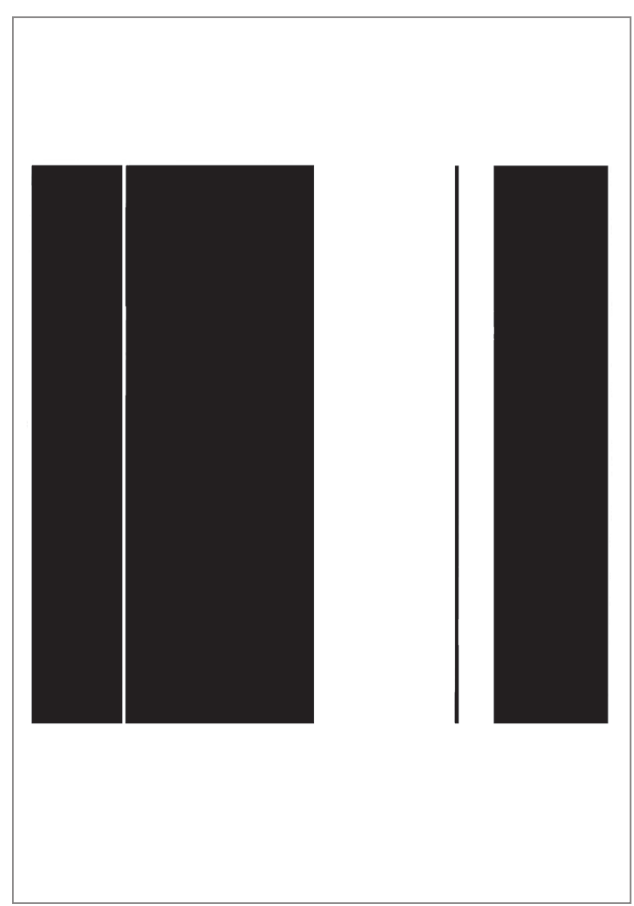

encuentra el verdadero carácter propedéutico del curso básico, el que preparará de forma óptima a los futuros Formgeber ${ }^{8}$ de la $\mathrm{HfG}$ para su actividad proyectual en los departamentos.

\section{Curso básico Bauhäusler}

El primer curso de la HfG comenzaría el 3 de agosto de 1953 y, en los meses previos, su director se encuentra inmerso en las cuestiones principales de la escuela: el proyecto del edificio en el Kühberg -una colina en el sur oeste de Ulm-, en la fundación del primer curso preliminar y la búsqueda de los futuros docentes 9 . Para este primer Grundkurs, Bill convoca a docentes de su confianza de los años de la Bauhaus que contribuyan con él a su refundación. Uno puede imaginar un primer año en el que se reencuentran en un nuevo claustro los antiguos docentes de la Bauhaus, cuando, en realidad, los cursos se concentraron de forma intensiva en varias semanas y la presencia de los Bauhäusler se sucedía con escasa coincidencia, debido a sus compromisos en Estados Unidos.

Walter Peterhans, antiguo profesor de fotografía llamado por Hannes Meyer a la Bauhaus, participa un único curso en el claustro de la HfG. En Ulm, Peterhans condensa en tres meses los dos cursos del visual training ${ }^{10}$ que está impartiendo en ese momento en el IIT de Chicago a los estudiantes de arquitectura del segundo y tercer año, bajo la dirección de Mies van der Rohe. La base filosófica y metodológica de las enseñanzas de Peterhans están asentadas en la idea platónica de que la belleza es una entidad universal y eterna que radica más que en el objeto, en su marco conceptual y en la persona que lo observa ${ }^{11}$. Con un carácter analítico, Peterhans propone diez ejercicios que profundizan por separado en las cualidades de la forma: proporción, color, textura, ocupación y sensación de espacio. En ellos se investigan las ilusiones ópticas del espacio, la relación de proporción entre líneas y rectángulos, la creación de texturas con objetos y la generación de ilusiones de espacio a través de la repetición y secuencias de línea, densidad, color y disposición de superficies planas. [1]

Nonné Schmidt, viuda de Joost Schmidt, es la Baushäusler que permanece más tiempo como docente en Ulm². Nonné es la única transmisora fiel que mantiene intacta la docencia de la etapa Bauhaus. Su didáctica se ajusta exactamente a las enseñanzas de la forma y el color de Klee y la teoría elemental de diseño de su marido, no en vano su curso se denominaba "Enseñanza de la forma y del color en Paul Klee y Joost Schmidt". [2]

Josef Albers viajaba a Ulm desde Yale donde era responsable del departamento de diseño. Nunca llega a ser un profesor permanente en la $\mathrm{HfG}$, pero tiene un peso importante durante los dos primeros cursos como docente y asesor en el currículum del curso básico. El curso de Albers para la HfG es el resultado de la recuperación de algunos de sus ejercicios de la Bauhaus y las nuevas investigaciones del color que está desarrollando en Norteamérica. Se vertebra en tres ejes: diseño básico, dibujo básico y color básico. 


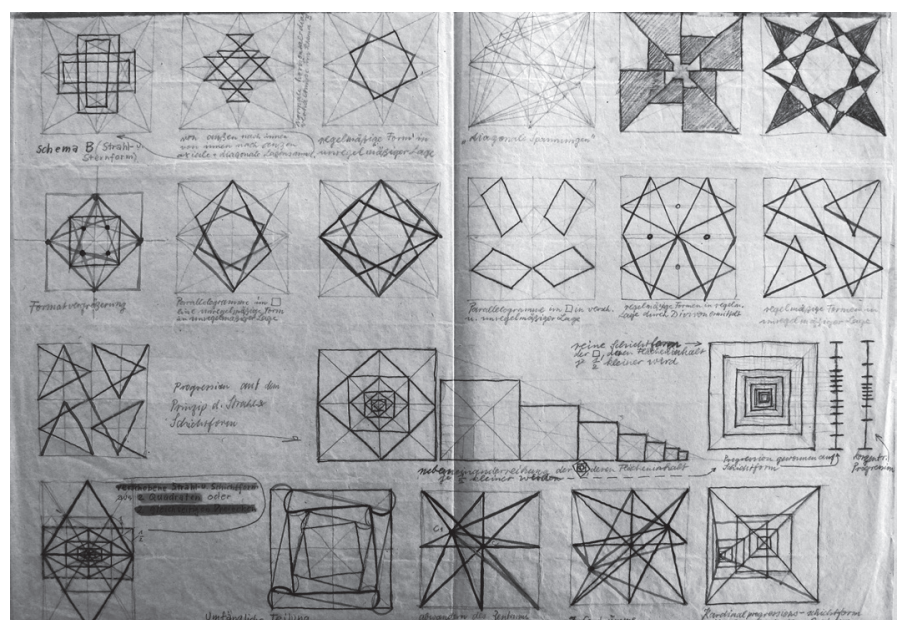

[2]

El diseño básico, herencia directa de la Bauhaus, introduce al alumno en la exploración del material y el descubrimiento de las posibilidades que ofrece ${ }^{13}$. Estos cursos se concentran fundamentalmente en la generación de formas con plegados de papel y manipulación de alambres, aunque de forma puntual también trabajó con otros materiales como los tensigrity de madera y cuerda, los plegados de acetatos y los dibujos en arena. El dominio del trazado a mano alzada se alcanzaba en el curso de dibujo básico, desarrollando de forma simultánea la destreza visual y la capacidad motora del alumno.

En el curso de color básico Albers continúa su enseñanza de color iniciada en Yale y contiene las cuestiones básicas que después publicará en Interacción del color (1963). Los ejercicios están basados en la ley Weber-Fechner y consisten en la interacción e interdependencia del color-color, color-forma, color-cantidad y color-disposición. De esta manera, Albers transmitía a sus alumnos el valor relativo del color, que depende de la interacción con su entorno. [3] Esta enseñanza del color representa un contrapunto a la que llevaba a cabo Nonné Schmidt. Albers promueve el trabajo con papeles de colores por ser un material más seguro y homogéneo que si se dependiera de la técnicas pictóricas y pigmentos donde la variación de los matices dificultaría el proceso.

El maestro Itten realiza una breve estancia de dos semanas en la $\mathrm{HfG}$ en la que pone en escena su retórica sobre el color y Max Bill formula ejercicios desde las premisas de arte concreto.

\section{Hacia un nuevo Formgeber}

En 1955, Tomás Maldonado ${ }^{14}$ llega a la HfG invitado por Max Bill y con él una transformación profunda se gesta en los dos cursos siguientes a su llegada y germina con la marcha de Bill en $1957^{15}$. En los cursos 55-56 y 56-57 las enseñanzas se organizan de manera que los alumnos adquieren una preparación previa antes de sumergirse durante tres meses en el Kurs Maldonado. Este planteamiento, de entrada, denota una mayor coordinación entre las enseñanzas y el soporte que ofrecen unas a otras. Este hecho es especialmente elocuente entre el Kurs Baravalle ${ }^{16}$ y el Kurs Maldonado. El Kurs Baravalle del 56-57 continúa la línea de cursos anteriores
[3]

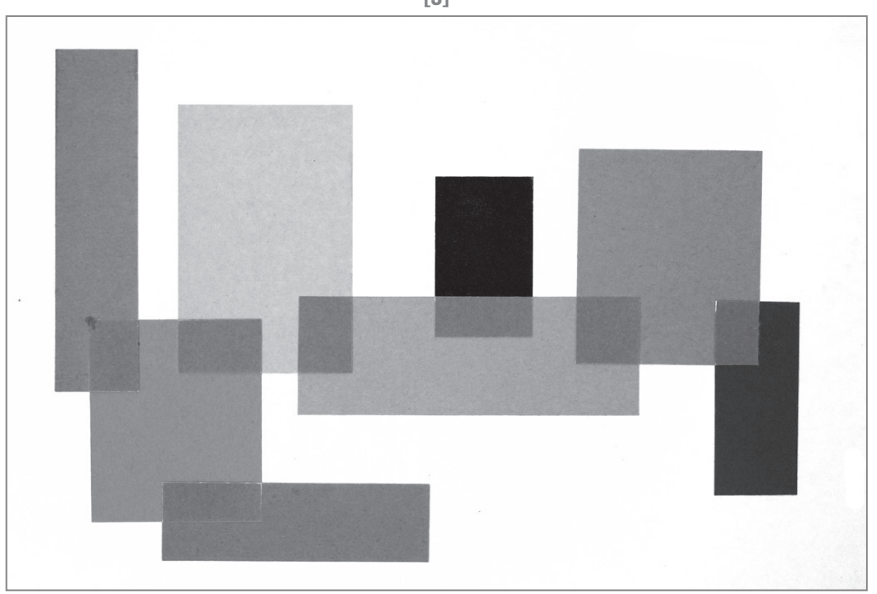

[4]

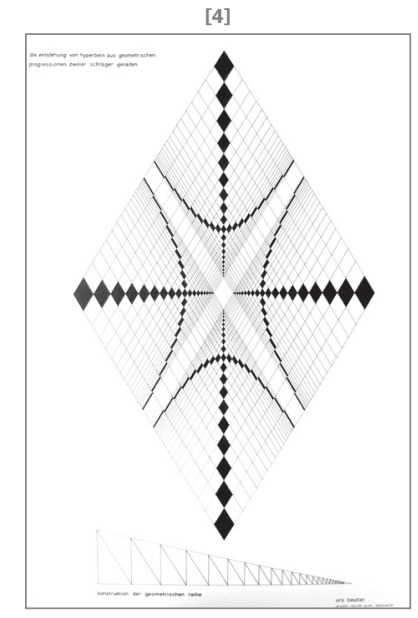

[2] "Estudios sobre el cuadrado. Enseñanza de la forma de Paul Klee". Curso Helene Nonné-Schmidt. Alumno Klaus Erler, curso 1953-54. Fuente: Archivo HfG Ulm.

[3] "Transparencia. llusión óptica. Obtención de la misma mezcla de color a partir de pares de colores muy diferentes". Curso de Color de Josef Albers. Alumno Christopher Naske, enero 1954. Fuente: Archivo HfG Ulm. las mediante la progresión geométrica entre pares de rectas oblicuas". Alumno Urs Beutler, Curso 1956-57. Fuente: Archivo HfG Ulm.

[5] Ejercicio del Visuelle Einfürung impartido por Tomás Maldonado. "Escala de blanco a negro en cuatro pasos, sobre un triángulo de Sierpinski". Alumno Peter Disch, noviembre 1955. Fuente: Archivo HfG Ulm.

[6] Ejercicio del Visuelle Einfürung impartido por Tomás Maldonado. "Negro como color". Alumna Meret Lindinger, enero 1956. Fuente: Archivo HfG Ulm.

[7] Ejercicio del Visuelle Einfürung impartido por Tomás Maldonado. "Precisión mediante imprecisión - imprecisión mediante precisión" Alumno Hans Von Klier, enero 1956. Fuente: Archivo HfG Ulm.
[4]. Kurs Baravalle. "Generación de hipérbo-

11 El Visual Training ha sobrevivido porque tiene una fuerte base filosófica y metodológica. El Visual Training se basa en el concepto platónico, reafirmado por Kant, por el que la Belleza está fundamentada en el sujeto más que en el objeto". DOMER, Denis E. "Walter Peterhans and the legacy of visual training". Reflections. Teaching architecture. The Journal of the school of architecture University of Illinois at Urbana-Champaing, $n^{\circ} 5$, otoño 1987, p. 23.

12 El motivo de la mayor presencia de Nonné Schmidt en la $\mathrm{HfG}$ fue porque tenía mayor disponibilidad, ya que residia en la cercana región del Lago Constanza, donde se ocupaba de la fundación de su marido.

13 "Para preparar el camino al descubrimiento y la invención, que son los indicadores de la creatividad, prefiero materiales poco conocidos o que normalmente no se usen para las formulaciones visuales. Estamos empleando los materiales de una manera en la que los estudiantes no habían pensado antes. Para evitar la mera puesta en práctica de la teoría y la técnica prefiero el método inductivo, es decir, llegar a conclusiones después de haber realizado los ejercicios, de haber adquirido experiencia". ALBERS, Josef. "Mis cursos en la Hochschule für Gestaltung de Ulm". Form, $n^{\circ} 4$, Cambridge: abril 1967, pp 8-10.

${ }^{14}$ El artista argentino, que había entrado en contacto con Max Bill para la publicación de una monografía sobre su obra, entró en la escuela en el año 55 como su ayudante. Al final del curso 55-56 compartía el rectorado de la escuela junto a Max Bill, Otl Aicher, Hans Gugelot y Vordemberge Gildewart. 
15 William S. Huff, alumno de la HfG y posteriormente profesor en Ulm y en la escuela de arquitectura de Buffalo, afirma que los cursos importantes de Maldonado fueron el 1955-56 -periodo de su formación como docente- $y$ el 1956-57 -año de su consolidación-. En estos dos cursos precedentes las enseñanzas del Grundkurs se organizan de forma que los alumnos tienen una preparación previa en la que adquieren una serie de destrezas antes de sumergirse durante tres meses en el Kurs Maldonado. HUFF, Williams. "Albers, Bill y Maldonado: el curso básico de la escuela de diseño de Ulm (HfG)" En: ROSENBERG, Adriana (ed). Tomás Maldonado, un itinerario. Milán: Skira, 2007, p. 104. Catálogo de la exposición celebrada en el Museo Nacional de Bellas Artes, Buenos Aires, noviembre 2007diciembre 2008.

${ }^{16}$ Hermann Edler von Brackenburg Baravalle nace el 27 de mayo de1898 en Viena (Austria-Hungria) y muere en Wiesneck in Buchenbach (Friburgo, Alemania) el 7 de junio de 1973, con 75 años. Educador, matemático, físico y astrónomo; director de la Sección Matemático-Astronómica en el Goetheanum de Dornach y profesor invitado en la HfG de Ulm entre los años 1954-1960 y 1967-1968.

17 MALDONADO, Tomás. "Rede des Vorsitzenden des Rektoratskollegiums der Hoschule für Gestaltung, Tomás Maldonado, zur Eröffnung des Studienjahres 1957/58 am Donnerstag den 3. Oktober 1957". (Discurso del Rector de la Escuela Superior de Diseño Hoschule, Tomás Maldonado, para la apertura del curso académico 1957-1958 en Jueves, de octubre 1957). Discurso inédito. HfG Ulm, 1957, p. 1.

${ }^{18}$ En este curso, además, se prestará especial atención a la formación matemática con el Dr. Eichhorn, así como a la introducción a la metodología impartida por la profesora Dra Walther. El Dr. Sperlich y el arquitecto Joseph Ryckwert dirigen el seminario histórico-cultural y el profesor Franzen la introducción a la sociología.

${ }^{19}$ Carta de Max Bill a Walter Gropius, 10 de febrero de 1959. SPITZ, René. HfG Ulm. The view venid the foreground. The political history of the Ulm school of design 1953-1968. Stuttgart-Londres: Alex Menges, 2002, p. 231

${ }^{20}$ MALDONADO, Tomás. "Bericht von Hern Tomás Maldonado in der Verwaltungsratsssitzung am 21 April 1958." (Informe de D. Tomás Maldonado en la reunión del consejo del 21 de abril de 1958). Discurso inédito. HfG Ulm, 1958 p. 4.

21 Horst Rittel enseñó un amplio rango de materias, incluyendo clases sobre metodología en todos los departamentos, MOA y en 196061 matemáticas, física y física técnica. En el departamento de diseño industrial enseñó mecánica general, en el departamento de comunicación visual, teoría de la información, en el departamento de Arquitectura, metodología, teoria de estructuras, introducción a la matemática lógica, permutaciones y combinaciones, topología, teoría de la ciencia y cibernética. REUTER, Wolf. “... den Dualismus zwischen ratonaler und intuitiver Tätigkeit auflösen" Horst Rittel an der HfG Ulm (“...disolviendo el dualismo entre la actividad intuitiva y racional"). Horst Rittel en la $\mathrm{HfG}$ Ulm. En VV.AA, op. cit. p. 94. basada en la geometría dinámica como generadora de la forma [4]. La representación de los elementos geométricos más sencillos -una recta- y sus posibles transformaciones, generalmente relacionadas con el movimiento -inversión-, tiene la intención de provocar una respuesta en cada alumno para generar otras formas -circunferencia-, de complejidad creciente. El Kurs Baravalle reactiva el sentido geométrico desde la percepción y la representación y conduce a la creación de un lenguaje de formas. Cualquier ejercicio queda abierto a nuevas posibles transformaciones.

Maldonado desarrolla unos ejercicios plásticos basados en una gramática visual sobre cuestiones no objetivas que pretende emplearse como respuesta a los problemas de diseño. Más que una introducción a la forma, la Visuelle Metodik pretendía constituir una metodología de trabajo. Para articular esta gramática, Maldonado se basa en ramas de la geometría próximas al arte concreto -curva de Peano, triángulo de Sierpinski, Curva de Weierstrass, simetría, parquetería-, así como a los problemas de figura fondo para los que la percepción de la psicología gestalt era una respuesta -el negro como color, exacto a través de inexacto, inexacto a través de exacto, efecto espacial, primer plano-fondo-. Para ambos docentes la geometría subyace en el planteamiento de sus ejercicios, no como un fin en sí mismo, sino como un soporte en el que se desarrollar el tema del ejercicio. [5] [6] [7]

Maldonado enuncia la ruptura definitiva con la Bauhaus en el discurso de apertura del curso 57-58 y, con ello, un cambio de rumbo: "Aceptamos solamente la actitud progresista y anti-convencional, el deseo de contribuir a la sociedad en su propia situación histórica. En este sentido, y solo en este sentido, estamos continuando la labor de la Bauhaus"17. En este discurso clave para comprender el rumbo que tomaría la escuela, Maldonado se lamenta que hasta el momento, la "racionalidad" por la que se empieza a conocer a la $\mathrm{HfG}$ es tan solo un disfraz científico. Anuncia que la escuela va a otorgar una mayor importancia a las asignaturas teóricas para la educación de un diseñador más modesto que conozca los materiales, las leyes de construcción y los procesos de fabricación con los que debe trabajar. De acuerdo a este objetivo, en el Grundkurs van a empezar a colaborar los profesores de los departamentos impartiendo docencia en el tercer cuatrimestre. Este, sin duda, es un adelanto de la aparición de las Metodische Übungen -tareas metodológicas-, el vuelco definitivo al enfoque propedéutico del curso básico ${ }^{18 .}$

Max Bill renuncia en 1957, augurando un futuro oscuro para la HfG. Está decepcionado con una escuela que se ve seducida por un "misticismo tecnológico"19 personificado en la figura de Maldonado. Este asume la dirección del Grundkurs y en marzo de 1958 se aprueban nuevos estatutos para la escuela en los que se establece una junta de gobierno de tres miembros: Maldonado, Aicher y Gugelot. Al finalizar el curso 57-58, Maldonado sigue impulsando su nueva formulación de la escuela a partir de los cambios científicos y tecnológicos de posguerra surgidos durante el conflicto con Max Bill y su marcha definitiva de la HfG. En su informe al consejo ${ }^{20}$, expone su creencia en un nuevo tipo de Formgeber que necesitará el planteamiento de los problemas y su análisis científico con herramientas metodológicas. Una de las herramientas que presenta Maldonado en este informe es el Análisis de Operaciones Matemáticas -MOA-, una disciplina desarrollada en EEUU que se sirve del método matemático para interpretar y analizar operaciones de cualquier tipo. La segunda asignatura sería teoría de la ciencia, que pretende sustituir la visión idealista del mundo por una de orientación científica. En estas nuevas materias se encuentra larvada una transformación del curso básico que tomaría otro rumbo nunca planeado por Maldonado.

\section{Acción planificadora}

La introducción del MOA en la HfG viene de la mano de una nueva figura determinante. Horst Rittel $^{21}$ llega como profesor invitado a la escuela con veintiocho años, recién licenciado en la universidad de Göttingen en Matemáticas puras y Física teórica. Del cuerpo docente de la HfG,
[5]

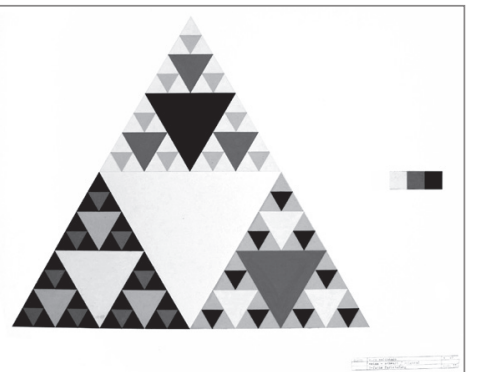

[6]

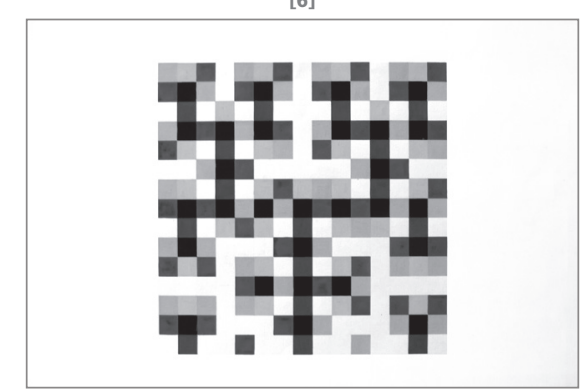

[7]

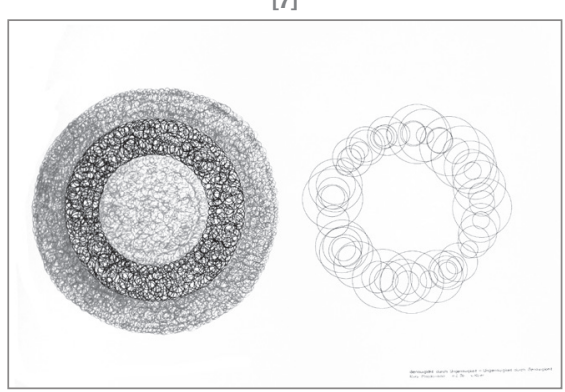




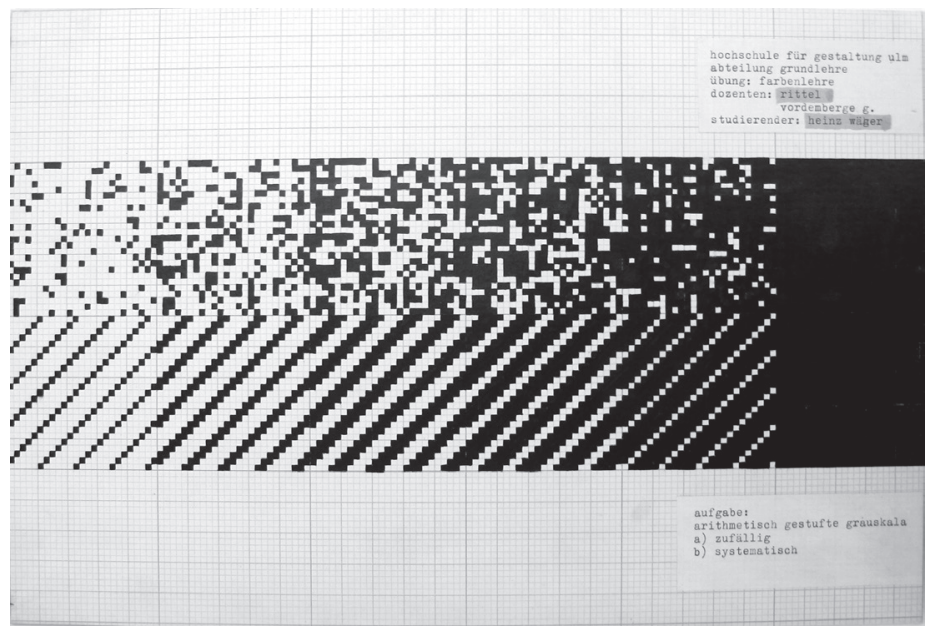

[8]
Rittel es el único verdadero hombre de ciencia -tras la marcha de Max Bense-, y por eso se le confían todas aquellas asignaturas, tanto en el Grundkurs como en los departamentos, que tuvieran que ver con metodología, matemáticas o física ${ }^{22}$. El objetivo de estas disciplinas era el de proporcionar un control del proceso de proyecto, una actividad que Rittel definió como acción planificadora.

Rittel adquiere una insólita influencia que le lleva a integrar una nueva junta de gobierno junto a Tomás Maldonado y Herbert Ohl a final del curso 58-59. En la conferencia pedagógica final de este mismo curso, Rittel impone, apoyado por la mayoría del claustro, una nueva configuración del curso básico en la que la metodología adquiere un peso definitivo. Maldonado vio frustrada su voluntad de continuar con su configuración ya establecida. Las lecciones de Rittel habían sido un mero apoyo teórico a su curso de gramática visual, y a partir de ahora se erigían como la subestructura que daría soporte a la propedéutica del proyecto. Como consecuencia, Maldonado se refugia en el Departamento de Comunicación Visual y no vuelve a intervenir en el curso básico.

En el curso 59-60 el bloque de Grundlehre estaba integrado por tres enseñanzas principales: el color, a cargo de Vordemberge-Gildewart, Fröshaug y Rittel, las Grafische Darstellung, dirigidas por Fröshaug, Rittel, Aicher y Schnaidt, y las Metodische Übungen, impartidas por profesores de los departamentos. En el bloque de medios de representación se sucedían las asignaturas de dibujo técnico, dibujo libre, escritura, expresión escrita, tipografía, teoría y práctica de la tipografía y trabajo de taller. El bloque científico se introduce por primera vez en el Grundkus con la física. En el bloque teórico Rittel imparte metodología y, además, los estudiantes tienen lecciones de historia cultural, política, fisiología, ergonomía, sociología y teoría de la percepción.

Centrémonos en el núcleo proyectual, el Grundlehre. El Farbenlehre -enseñanza de color- seguía incidiendo en escalas de grises, gradación de color e interacción pero con un marcado enfoque metodológico. Como ejemplo, Rittel y Vordemberge-Gildewart proponían la generación de una escala de grises, graduada aritméticamente desde el blanco al negro, mediante la ocupación de las celdas de una retícula de manera progresiva en dos distribuciones paralelas: aleatoria y sistemática. [8]
[8] Curso de color de Horst Rittel y Friedrich Vordemberge-Gildewart. Dos escalas de grises graduadas aritméticamente; ocupación aleatoria y sistemática. Alumno Heinz Wäger, curso 1959-60. Fuente: Archivo HfG Ulm.

[9] Grafische Darstellung, ejercicio dirigido por Claude Schnaidt. Representación de las secuencias del movimiento de un ama de casa en su vivienda a lo largo del dia. Alumnos Jan Wilke Beenker y Robert Graeff, abril 1960. Fuente: Archivo HfG Ulm.

[9]

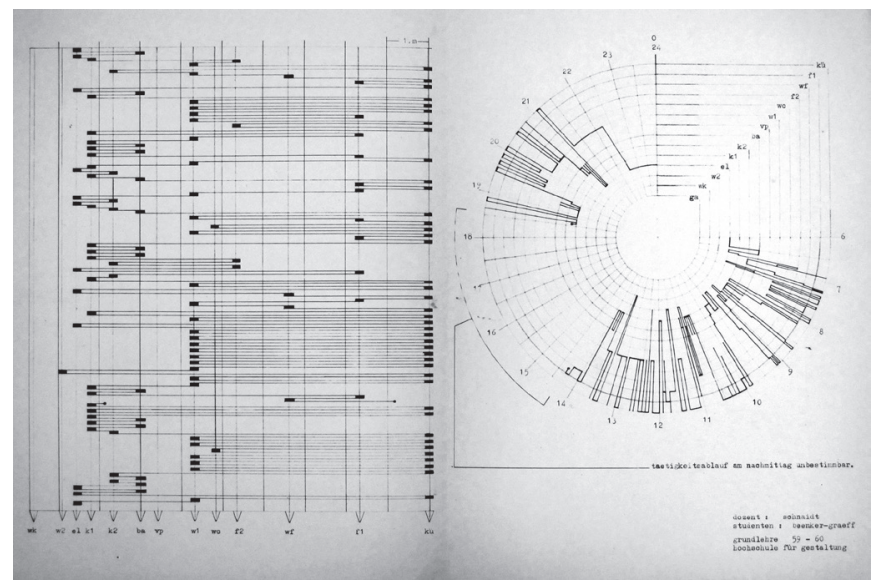




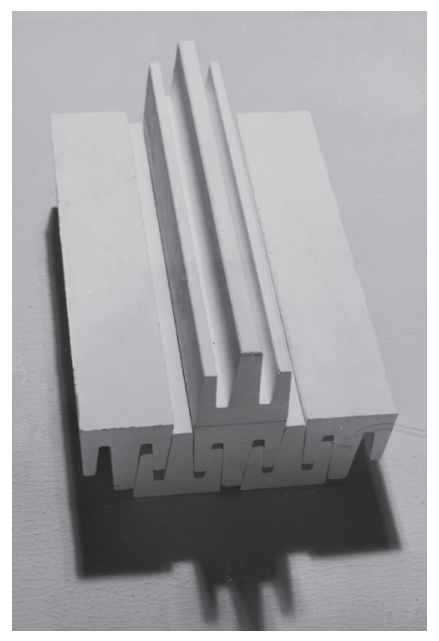

[10]

[10] El mismo enunciado da lugar a respuestas muy diversas. Methodische übung $\mathrm{n}^{\circ} 4$. Tarea metodológica dirigida por Walter Zeischegg. Diseño de un lingote conformado y apilable de aluminio en bruto. Fotografia de las maquetas en yeso. Alumno: Walter Eichenberger, noviembre 1959. Fuente: Archivo HfG Ulm.

[11] El mismo enunciado da lugar a respuestas muy diversas. Methodische übung $n^{\circ} 4$. Tarea metodológica dirigida por Water Zeischegg. Diseño de un lingote conformado y apilable de aluminio en bruto. Planos. Alumno Robert Graeff, curso 1959-60. Fuente: Archivo HfG Ulm.

[12] Methodische übung $n^{\circ} 1$. Tarea metodológica dirigida por Anthony Froshaug. Reloj luminoso con indicaciones horarias variables. Alumno Walter Eichenberger, noviembre 1959. Fuente: Archivo HfG Ulm.

[13] Methodische übung $\mathrm{n}^{\circ}$ 1. Tarea metodológica dirigida por Anthony Froshaug. Reloj luminoso con indicaciones horarias variables. Alumno Robert Graeff, noviembre 1959.

Fuente: Archivo HfG Ulm.

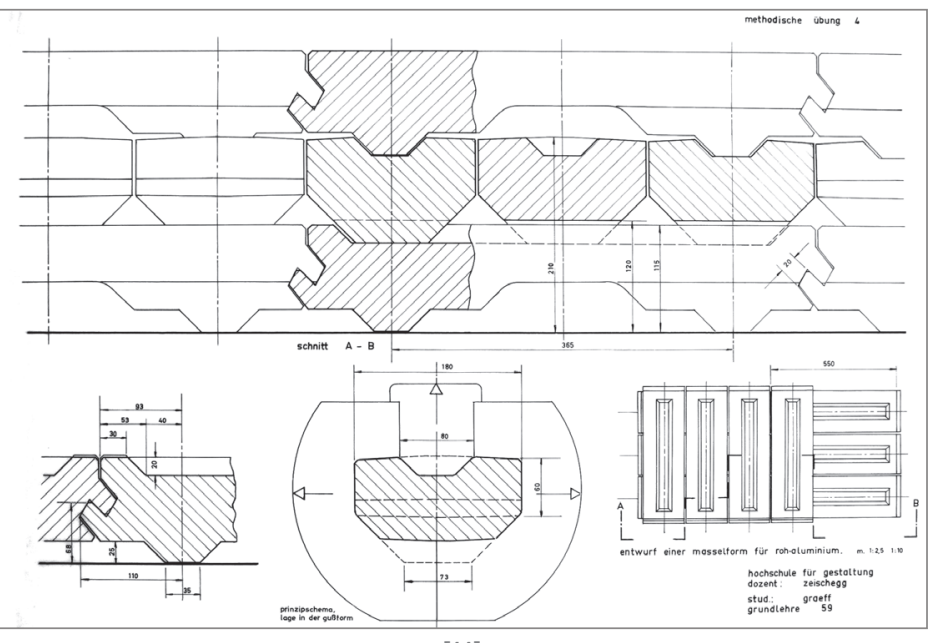

[11]

Las Grafische Darstellungen -representaciones gráficas- se imparten como un método analítico y de conceptualización visual, mediante el desarrollo de diagramas. Los alumnos adquieren la capacidad de analizar y sintetizar la información para la representación gráfica de los focos de un problema. Tick-tack-toe-spiel, tercer ejercicio dirigido por Fröshaug y Rittel, consiste en el desarrollo y representación de la combinatoria contenida en las reglas del juego de las tres en raya. El séptimo ejercicio, propuesto por Claude Schnaidt, se centra en la representación de las secuencias de movimiento de la rutina diaria de un ama de casa en el interior de su vivienda [9]. Los diagramas que elaboran los estudiantes tienen que contener los cambios de habitación, su frecuencia y la longitud de los caminos recorridos entre las diferentes estancias. El repertorio de soluciones gráficas es muy rico y variado, a pesar de que todos los ejercicios parten del mismo enunciado.

Las Metodische Übungen -tareas metodológicas- encarnan, mejor que ningún otro ejercicio, la acción planificadora que enunciaba Rittel. A lo largo del 59-60, los alumnos del Grundkurs desarrollaban dieciséis pequeños proyectos dirigidos por seis profesores procedentes de los departamentos: comunicación visual -Fröshaug-, diseño industrial -Gugelot y Zeischegg-y construcción -Herbert Ohl-. Los profesores se alternaban en las enseñanzas y, para planificar y coordinar esta secuenciación, celebraban reuniones pedagógicas.

El planteamiento de los proyectos seguía el mismo esquema en todos los casos. El profesor repartía un enunciado a los estudiantes. Este enunciado, en casos como el de Fröshaug, consistía en un guión que pautaba el itinerario generador de las condiciones del proyecto. Se pedía a los alumnos que registraran las fases en el desarrollo de las soluciones y que elaboraran un informe final sobre todo el trabajo, que debían entregar junto con la propuesta gráfica y las maquetas.

Cada una de las dieciséis Metodische Übungen entraña una singularidad específica del área proyectual al que pertenece. Las que dirige Zeischegg -núm.4 diseño de lingotes de aluminio, núm. 7 cubos apilables, núm. 11 cuerpo de rotación/cuerpo de penetración y núm.15 rueda de trinquete- se formulan desde el ámbito del diseño industrial. Las soluciones de los estudiantes

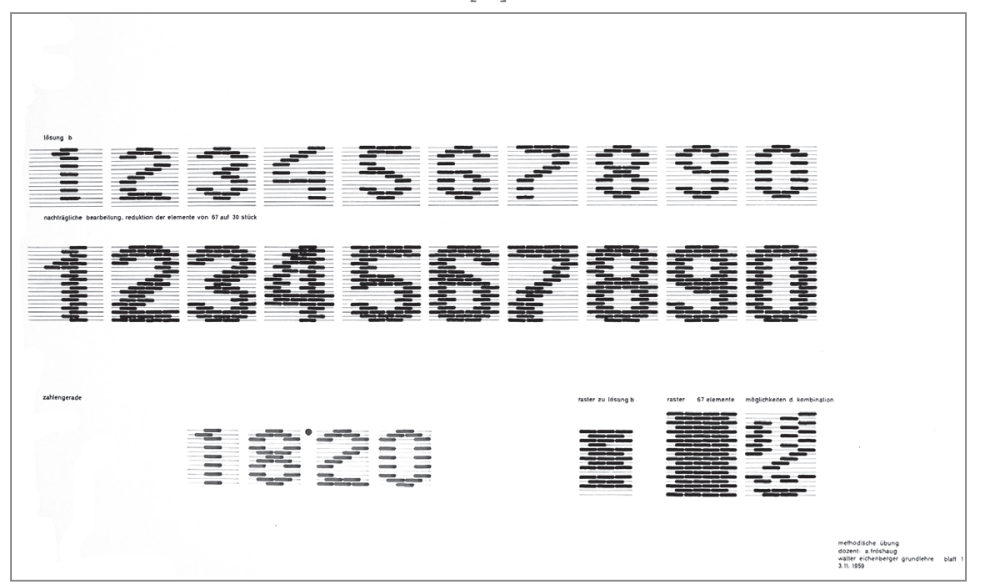

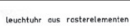

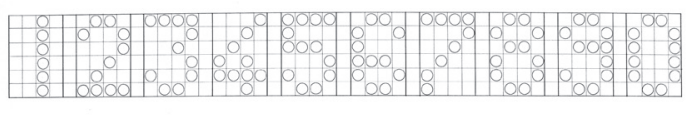

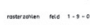

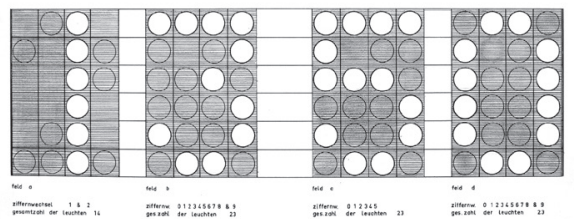




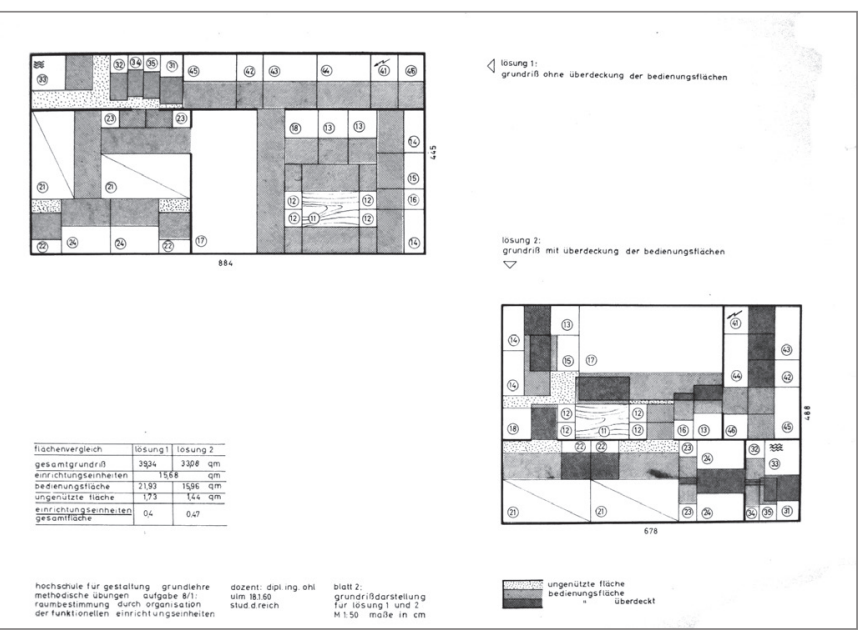

[14]

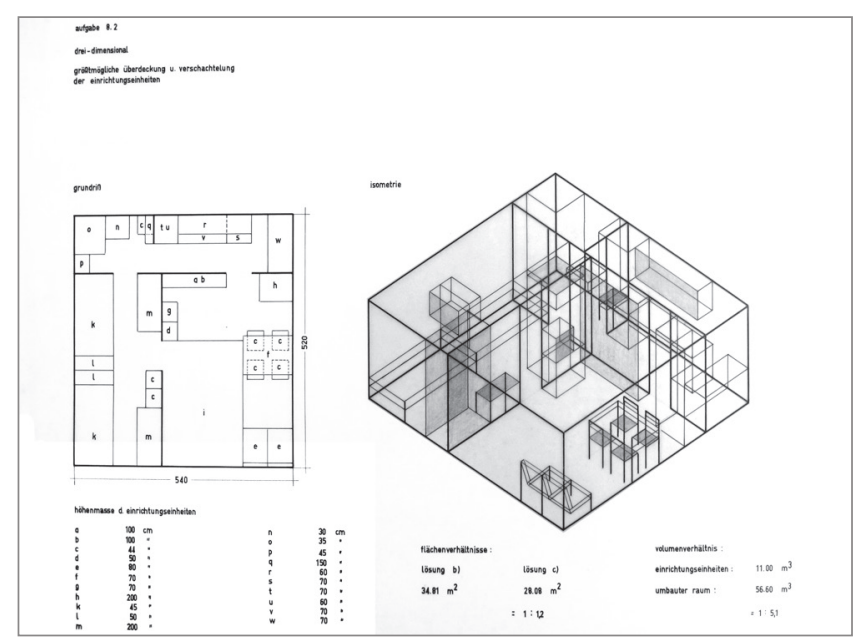

[15] están basadas en la geometría como generadora de la solución. De estas propuestas se desprende un fuerte vínculo entre el desarrollo gráfico y su construcción en maquetas de yeso. [10] [11]

Fröshaug propone proyectos desde el ámbito de la comunicación visual -núm.1: reloj luminoso con indicaciones horarias variables [12] [13], núm.2: diseño del indicador de llegadas de una estación de tren con dígitos legibles por humanos y máquinas, núm.3: diseño para un juego de pesas, núm.13: formato de composición para una revista, núm.14: representación de las relaciones de comunicación en el edificio de la HfG-. Fröshaug abraza con entusiasmo esta nueva formulación del curso básico en el que, al fin, las enseñanzas generan una urdimbre de conocimientos al servicio del proyecto: "El cambio fue saludable: por primera vez, las diferentes tareas estaban coordinadas entre sí y con el trabajo en los seminarios. Para aquellos problemas como el diseño del indicador de llegadas de una estación de tren con dígitos legibles por humanos y máquinas, tuve la posibilidad de referirme a las lecciones de Perrine en percepción, a las lecciones de Rittel de combinatoria, a varias consideraciones de requisitos económicos basadas en las conferencias de sociología de Kesting, y también fue útil saber qué tarea sucedería a la mía"23.

Herbert Ohl, del departamento de arquitectura, dirige un ejercicio denominado "estimación del espacio determinado mediante la organización de unidades de equipamiento". Los estudiantes deben explorar diferentes definiciones de un espacio que surge de las relaciones métricas y topológicas entre unas unidades funcionales dadas. Las unidades funcionales son muebles y elementos de servicio vinculados a un espacio de servidumbre. Ohl propone dos itinerarios de trabajo: sin solapamiento -las unidades funcionales independientes de los espacios servidos- y con solapamiento entre las superficies de servidumbre. Las dos soluciones deben responder a unos objetivos impuestos en el enunciado: espacios mínimos, extensión superficial más baja, formas compactas, acceso simultáneo a todas las unidades y trayecto total mínimo. [14] [15]

Los proyectos que se desarrollaban en las Metodische Übungen partían de unas condiciones rigurosas con un margen de trabajo delimitado. Este es el caso del ejercicio núm.12 de Hans Gugelot. Los alumnos debían afrontar un problema constructivo muy acotado: diseñar el bastidor de soporte para una esfera de $10 \mathrm{~cm}$. de diámetro, manipulando una lámina cuadrada de hojalata de $20 \mathrm{~cm}$. de lado y $3 \mathrm{~mm}$. de espesor. A pesar de lo específico del enunciado, los estudiantes desarrollan propuestas muy diferentes entre sí. [16] [17]

\section{Propedéutica del proyectar}

Tras el cierre de la Bauhaus, el Vorkurs que allí se impartía era una suerte de curso básico legendario que numerosas escuelas de arte y arquitectura en Europa y América de mitad del siglo XX trataban de rescatar. Lo cierto es que hubo tantas versiones como personalidades que lo abordaron, desde la expresionista de Itten a la racionalista de Albers. Esta desorientación es la que asume en un primer momento la HfG. En su fundación, tiene una mayor importancia el efecto llamada de los protagonistas de la Bauhaus, y la legitimidad que a través de ellos quiere alcanzar la nueva escuela, que las enseñanzas en sí. En la HfG esta refundación del curso básico de la Bauhaus no implica una continuación en la metodología de la escuela alemana: los antiguos Bauhäusler han pasado algunos años como profesores en Estados Unidos y han evolucionado en su manera de enseñar. Las lecciones de los consagrados maestros en Ulm son en mayor medida una síntesis de sus experiencias norteamericanas más que un revival de la Bauhaus. Los
[14] Methodische übung $\mathrm{n}^{\circ} 8$. Tarea metodológica dirigida por Herbert Ohl. Espacio determinado mediante la organización de unidades de equipamiento. Alumno Dieter Reich, enero 1960. Fuente: Archivo HfG Ulm.

[15] Methodische übung $\mathrm{n}^{\circ}$ 8. Tarea metodológica dirigida por Herbert Ohl. Espacio determinado mediante la organización de unidades de equipamiento. Alumno Heinz Wäger, enero 1960. Fuente: Archivo HfG Ulm

[16] Methodische übung $\mathrm{n}^{\circ}$ 12. Tarea metodológica dirigida por Hans Gugelot. Bastidor de soporte para una esfera de $100 \mathrm{~mm}$ de diámetro. Material: lámina de hojalata de 200 $\mathrm{mm} \times 200 \mathrm{~mm}$ y 0,3 mm de espesor. Alumno Peter Beck, marzo 1960. Fuente: Archivo HfG Ulm.

[17]. Methodische übung $\mathrm{n}^{\circ}$ 12. Tarea metodológica dirigida por Hans Gugelot. Bastidor de soporte para una esfera de $100 \mathrm{~mm}$ de diámetro. Material: lámina de hojalata de 200 $\mathrm{mm} \times 200 \mathrm{~mm}$ y $0,3 \mathrm{~mm}$ de espesor. Alumno Robert Graeff, marzo 1960. Fuente: Archivo HfG Ulm. 


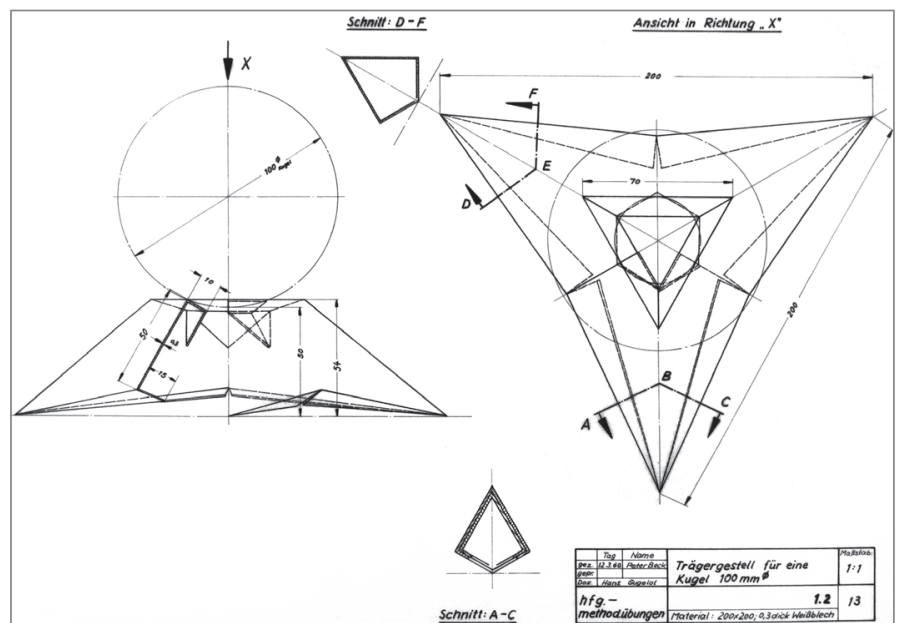

[16]

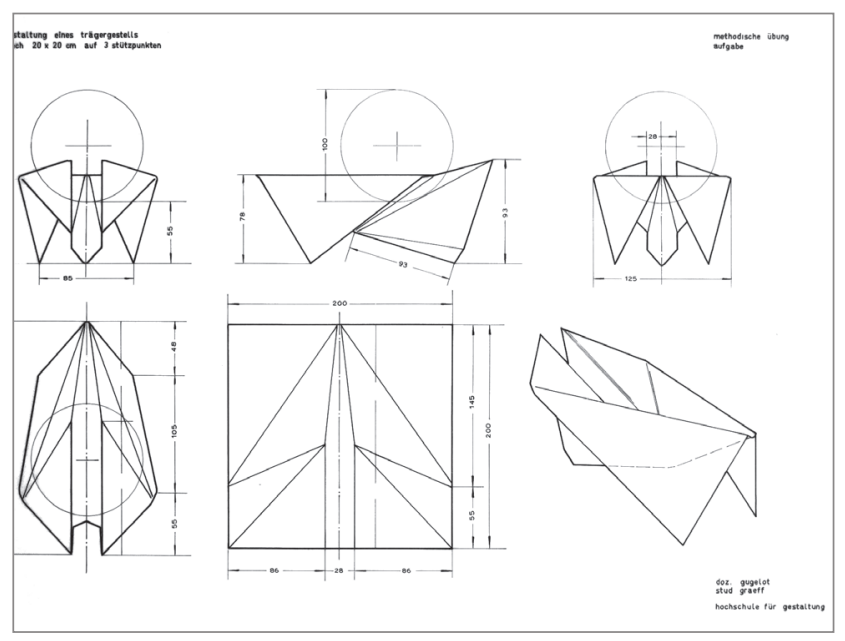

[17] $\mathbf{2 3}$ "Lamentablemente, esa conquista ha sido más leyenda que realidad. Tras el cierre abrupto de la Bauhaus en 1933, las ideas de la escuela se diluyeron y dispersaron por el mundo. De vasto alcance pero superficial: tal fue el especial destino del curso preliminar (...) El problema fue que hubo múltiples versiones de él: la versión expresionista-psicologista de Itten, la versión experimental excesvamente simplista de Moholy-Nagy, la versión racionalista de "ojos abiertos" de Albers. Más aún, la enseñanza impartida por Kandinsky y Klee, catalogada como parte de una Teoría de la Forma (Formlehre), obligatoria, era una especie de compendio de la infinidad de cambios que sucedieron posteriormente". En: ROSENBERG, Adriana, (op. cit), p. 104 primeros alumnos reciben una preparación que descansa en mayor medida en la representación, la creatividad y el lenguaje visual que en el desarrollo del pensamiento sobre el proyecto. La propedéutica en esta fase es la sucesión de diferentes didácticas sobre la forma y sus leyes visuales.

Maldonado reorienta el objetivo enunciando el tipo de diseñador y arquitecto que tiene que educar la escuela. En consecuencia, las enseñanzas se transforman bajo este nuevo destino. Se atisba un principio de coordinación entre las materias y estas adquieren por primera vez en la HfG un carácter propedéutico, es decir preparatorio para las disciplinas que se van a enseñar más tarde. La Visuelle Einführung de Maldonado se imparte tras unas materias que preparan al alumno para su asignatura. Los cursos se suceden como en la época bauhäusler, pero esta vez con una estructura planificada que persigue unos objetivos concretos. Maldonado cuenta con tres años consecutivos para definir un programa de tareas que perfila y depura progresivamente. Las tareas no se proponen desde el análisis de las cuestiones visuales sino que tienen un carácter propositivo: el alumno debe ofrecer una respuesta a un enunciado. El ejercicio "el negro como color" aborda la composición de una trama cromática en la que el negro tiene que interactuar con los demás colores de forma que no sea percibido como un vacío sino como un color más dentro de la composición. En la tarea "precisión a través de medios imprecisos" el objetivo es definir una forma plástica concreta mediante la imprecisión de la técnica gráfica, como por ejemplo, estampaciones con tela, aplicación de pigmentos con pincel seco o garabatos. Sus ejercicios evolucionan para conformar un curso coherente en el que incorpora en los ejercicios visuales cuestiones matemáticas y psicológicas -topología, percepción-, como elementos guía para la solución de los enunciados. De este modo, el Kurs Maldonado no solo ofrece una introducción visual, sino una sistematización de las acciones en el proceso de proyectar la solución, una pre-metodología.

Es en las Methodische Übungen donde la didáctica del curso básico alcanzará un carácter preparatorio más evidente, tanto en la propuesta de las tareas como en su proceso de proyecto: los enunciados plantean la ejecución de propuestas sobre temas propios de los departamentos de diseño industrial, comunicación visual o construcción industrializada. La metodología, en este caso incorporada en el proceso de análisis de la información, proporcionará así mismo las herramientas para abordar la ejecución de la solución. Sin embargo, la concreción en un objeto específico como tema de proyecto y la prevalencia de las herramientas metodológicas, conlleva la pérdida de la exploración mediante las variables abstractas y generales que proporcionaban las versiones anteriores del curso básico. Las destrezas visuales y las capacidades de generación de la forma desde un enfoque más universal quedan descartadas.

Tras este recorrido, cabe plantearse si la propedéutica del proyectar debe estar conformada por un rango de enseñanzas complementarias, que proporcionen al estudiante las destrezas necesarias para enfrentarse a un acercamiento al proceso en todas sus fases, desde todos los condicionantes y asumiendo distintos papeles. ¿Qué tipo de preparación favorece un enfoque panorámico -que permita contemplar el conjunto del proceso- y a la vez panóptico -que no pierda la perspectiva global del problema desde cada enfoque particular-? La metodología estricta para resolver problemas particulares no sustituye los procesos de pensamiento e ideación abstractos. El Grundlehre de la HfG, en su corto pero intenso recorrido de ocho años, contiene suficientes planteamientos válidos en sí mismos como para formular estructuras en las que puedan confluir las enseñanzas. 